\title{
Structured Analysis of Arbitrary Island Grids
}

\author{
Markus Jostock, Jürgen Sachau, Jean-Régis Hadji-Minaglou, University of Luxembourg \\ Christian Tuttas, University of Kaiserslautern
}

\begin{abstract}
This paper recapitulates an analytic control model for arbitrary island grids and presents findings on the dynamic behaviour of purely inverter driven island grids. Pole zero plots of different grid structures are presented and the influence of several inverter parameters on the grid stability is analysed. Findings show that for improved grid stability the inverter's time constants should also relate to their rated power. Further it is shown how $P-Q$ rotation in the droop control can improve the integration density of renewable power sources in the distribution grid.
\end{abstract}

Index Terms-Control Model, Island Grids, Inverters, Droop Control, Grid Stability

\section{INTRODUCTION}

The management of islanded power grids, detached from the interconnected European grid, becomes an important option for load management and recovery of black outs. If e.g. a sub-grid has sufficient renewable energy resources available, there is no reason to let the connected customers suffer from a blackout. Likewise, remote settlements may be operated in island mode, thus avoiding the installation of costly medium voltage lines.

The model [1] used in this paper has been developed in order to judge the stability of such island grids [2], even without rotating synchronous generators and their slowed interactions due to the mechanical inertias[3]. Previous research for smaller grids is presented in [4][5] and [6].

In island mode, particularly inverters cannot operate at maximum power point, but need to feed in exactly the currently consumed energy. All generators operate in droop control, where they behave like controlled AC voltage sources. The presented control model is suitable for any type of generator, acting as voltage source in the grid. While it was designed for island grid analysis, also structurally known part-grids at weak connection points can be modelled. It is based on the linearised power equations and is constructed with an analytical approach, beyond iterative numeric approximations. Thus it provides comprehensive access to the overall system's poles and zeros, allowing for stability analysis and design of robust controls. The model can comprise any number of generators and address arbitrary grid structures, without the need for repetitive manual construction of the differential equations.

The paper presents insights in the dynamics of inverter driven micro grids, revealed by the presented model. It shows the dependency of grid stability on several inverter parameters like time constants, rated power, as well as on grid parameters and line characteristics.

\section{ARBITRARY GRID MODEL}

The presented model is a linear, time-invariant (LTI) control model with multiple inputs and multiple outputs (MIMO). In order to capture an arbitrary grid with a potentially very high number of (small) generators, the model is split in two parts:
1) $w$ grid nodes where generators are connected, acting as dynamic AC voltage sources,

2) $l$ passive grid nodes where consumers are connected and power routing depends on the grid structure.

The model separation is depicted in figure 1 .

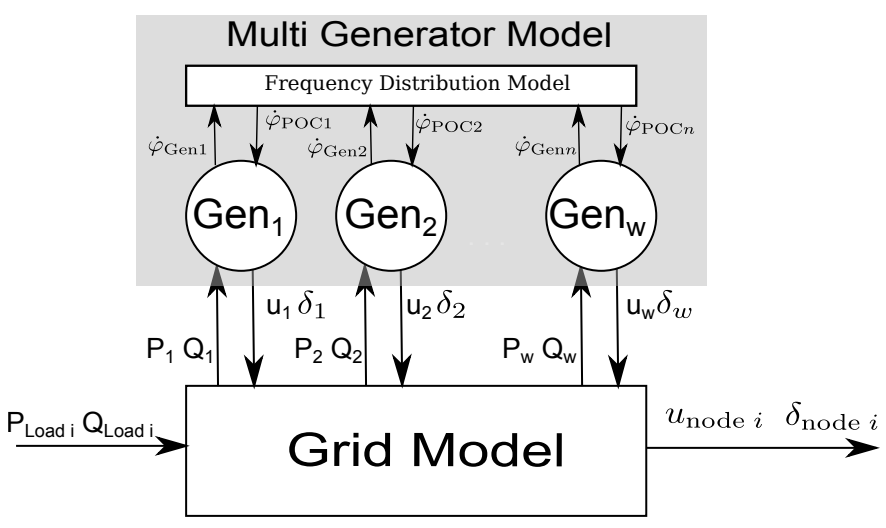

Figure 1. Separation of generators and passive grid

The generators are droop controlled AC voltage sources. Their respective output parameters are the voltage phase angle $\delta$, depending on the currently injected active power $P$, and the voltage amplitude $u$, depending on the actually provided reactive power $Q$.

\section{A. Multi Generator Model}

The design of the multi inverter model starts with a state space model of a single inverter, based on a droop controlled generator model from [7] and [8], as depicted in figure 2.

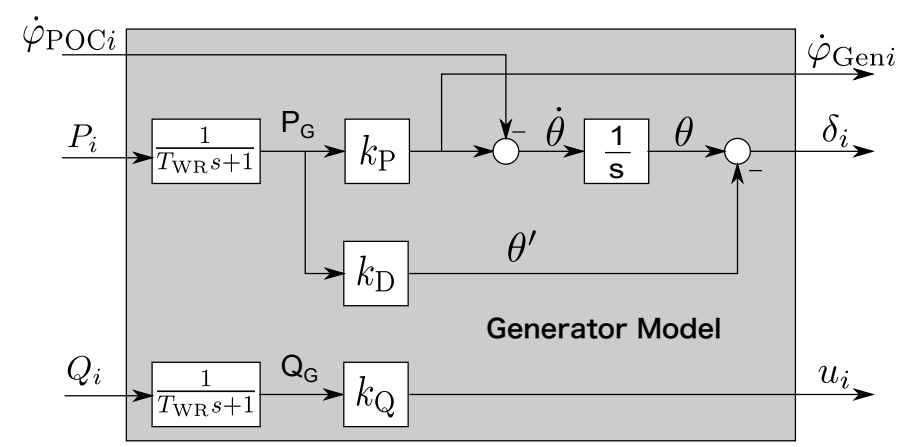

Figure 2. Control Model of a Single Inverter

A $3^{\text {rd }}$ order state space model is derived for the $i^{\text {th }}$ inverter in the form $\dot{x}_{i}=\mathcal{A}_{i} x_{i}+\mathcal{B}_{i} u_{i}, y_{i}=\mathcal{C}_{i} x_{i}$, with droops $k_{P}$ and $k_{Q}$, damping $k_{D}$. The state vector is defined as $\vec{x}_{i}=$ $\left[P_{G}, Q_{G}, \theta\right]_{i}^{T}$ and the input vector is $\mathfrak{u}_{i}=\left[P, Q, \dot{\varphi}_{\mathrm{POC}}\right]_{i}^{T}$, as displayed in figure 2 : 


$$
\begin{aligned}
& \frac{d}{d t} \vec{x}_{i}= {\left[\begin{array}{ccc}
-\frac{1}{T_{\mathrm{WR}}} & 0 & 0 \\
0 & -\frac{1}{T_{\mathrm{WR}}} & 0 \\
k_{\mathrm{P}} & 0 & 0
\end{array}\right] \vec{x}_{i}+\left[\begin{array}{ccc}
\frac{1}{T_{\mathrm{WR}}} & 0 & 0 \\
0 & \frac{1}{T_{\mathrm{WR}}} & 0 \\
0 & 0 & -1
\end{array}\right] \mathfrak{u}_{i} } \\
& {\left[\begin{array}{c}
\delta \\
u \\
\dot{\varphi}_{\mathrm{VSI}}
\end{array}\right]_{i}=\left[\begin{array}{ccc}
-k_{D} & 0 & 1 \\
0 & k_{Q} & 0 \\
k_{P} & 0 & 0
\end{array}\right] x_{i} }
\end{aligned}
$$

$\dot{\varphi}_{\mathrm{Gen} i}$ is the generator's injection frequency. $\dot{\varphi}_{\mathrm{POC} i}$ is the grid frequency at the $i^{\text {th }}$ generator's point of connection (POC), produced by all other generators in the gird. It is used to calculate the drift angle $\theta$. The power input $\vec{s}_{i}=\left[P_{i}, Q_{i}\right]^{T}$ and the voltage output $\vec{\nu}_{i}=\left[\delta_{i}, u_{i}\right]^{T}$ are separated from the frequency variables $\dot{\varphi}_{\mathrm{VSI}}$ and $\dot{\varphi}_{\mathrm{POC}}$. The reason is, that in a purely inverter driven power grid each inverter adapts its injection frequency $\dot{\varphi}_{\text {VSI }}$ with high dynamics. During transient phases there is no central grid frequency, but in each point of connection, temporarily a different frequency $\dot{\varphi}_{\mathrm{POC}}$ is valid. The generator model separates this frequency aspect from the power flow aspects. In order to capture all active generators in the grid, all single inverter state space models are block-diagonally composed into large dynamic system matrices. All generator input vectors are stacked to $\vec{s}_{\mathrm{Gen}}=$ $\left[\begin{array}{lllll}\vec{s}_{1}^{T} & \vec{s}_{2}^{T} & \vec{s}_{3}^{T} & \cdots & \vec{s}_{w}^{T}\end{array}\right]^{T}$ and all their output voltage vectors are stacked as $\vec{\nu}_{\text {Gen }}=\left[\begin{array}{lllll}\vec{\nu}_{1}^{T} & \vec{\nu}_{2}^{T} & \vec{\nu}_{3}^{T} & \cdots & \vec{\nu}_{w}^{T}\end{array}\right]^{T}$. The multi generator model exchanges only the power vector $\vec{s}$ and the voltage vector $\vec{\nu}$ with the subsequently described network model, as depicted in figure 1.

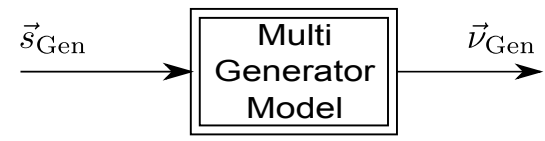

Figure 3. Multi Generator Model Block

\section{B. Grid Model}

In order to integrate the complete grid structure into the compound model, the node incidence matrix $\mathcal{K}$ is formulated. It contains the network information as representation of a directed network graph. $\mathcal{K}$ states which of the $n$ nodes are connected through the $t$ lines (twigs), whilst indicating the direction of positive power flow.

The node incidence matrix is sorted: All $w$ generator nodes are on top, all $l$ passive grid nodes are at the bottom. This allows for partitioning into the upper matrix $\mathcal{K}_{\mathrm{G}}$ containing generator nodes and the lower matrix $\mathcal{K}_{\mathrm{L}}$ containing the load nodes. The diagonal matrix $\mathcal{Y}=\operatorname{diag}\left(y_{1}, y_{2}, \ldots, y_{t}\right)$ contains the admittances value $y_{i}$ of each of the $t$ lines. It is used to calculate the power flows through the grid. The vector of

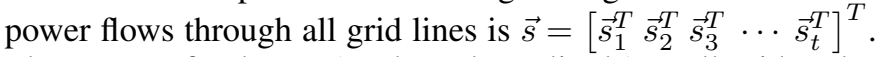
The vector of voltages (angle and amplitude) at all grid nodes is $\vec{\nu}=\left[\begin{array}{lllll}\vec{\nu}_{1}^{T} & \vec{\nu}_{2}^{T} & \vec{\nu}_{3}^{T} & \cdots & \vec{\nu}_{l}^{T}\end{array}\right]^{T}$. It can be split into the generator voltages $\vec{\nu}_{\mathrm{Gen}}$ and the load node voltages $\vec{\nu}_{\mathrm{Load}}$. The power flows through $t$ grid lines can be calculated with

$$
\begin{aligned}
\vec{s} & =-\mathcal{Y} \mathcal{K}^{T} \cdot \vec{\nu} . \\
& =-\mathcal{Y}\left[\begin{array}{ll}
\mathcal{K}_{G}^{T} & \mathcal{K}_{L}^{T}
\end{array}\right]\left[\begin{array}{c}
\nu_{\text {Gen }} \\
\nu_{\text {Load }}
\end{array}\right]
\end{aligned}
$$

The horizontally partitioned matrix $\mathcal{K}$ can also be used to formulate the power flow balance: all injected powers $\vec{s}_{\text {Gen }}$ and all loads $\vec{s}_{\text {Load }}$ drained from the grid equal the sum of the twig power flows:

$$
-\left[\begin{array}{c}
\vec{s}_{\text {Gen }} \\
\vec{s}_{\text {Load }}
\end{array}\right]=\left[\begin{array}{c}
\mathcal{K}_{G} \\
\mathcal{K}_{L}
\end{array}\right] \cdot \vec{s}
$$

Inserting the twig flows $\vec{s}$ from equation (2) into equation (4) leads to

$$
\left[\begin{array}{c}
\vec{s}_{\text {Gen }} \\
\vec{s}_{\text {Load }}
\end{array}\right]=\left[\begin{array}{cc}
\mathcal{K}_{\mathrm{G}} \mathcal{Y} \mathcal{K}_{G}^{T} & \mathcal{K}_{\mathrm{G}} \mathcal{Y} \mathcal{K}_{\mathrm{L}}^{T} \\
\mathcal{K}_{\mathrm{L}} \mathcal{Y} \mathcal{K}_{\mathrm{G}}^{T} & \mathcal{K}_{\mathrm{L}} \mathcal{Y} \mathcal{K}_{\mathrm{L}}^{T}
\end{array}\right] \cdot\left[\begin{array}{c}
\vec{\nu}_{\mathrm{Gen}} \\
\vec{\nu}_{\text {Load }}
\end{array}\right]
$$

Rearranging equation (5) leads to a matrix form where all known or computable variables are on the right hand side and the unknown variables $\vec{s}_{\text {Gen }}$ and $\vec{\nu}_{\text {Load }}$ are on the left hand side:

$$
\left[\begin{array}{c}
\vec{s}_{\text {Gen }} \\
\vec{\nu}_{\text {Load }}
\end{array}\right]=\left[\begin{array}{cc}
\mathcal{M}_{\mathrm{A}} & \mathcal{M}_{\mathrm{B}} \\
\mathcal{M}_{\mathrm{C}} & \mathcal{M}_{\mathrm{D}}
\end{array}\right] \cdot\left[\begin{array}{c}
\vec{\nu}_{\text {Gen }} \\
\vec{s}_{\text {Load }}
\end{array}\right] .
$$

Vector $\vec{s}_{\text {Load }}$ is defined as compound system input and is thus known. Vector $\vec{\nu}_{\text {Gen }}$ can be calculated as output of the multi generator model if the generator powers $\vec{s}_{\text {Gen }}$ are known. The equation system 6 can be solved with linear algebra and the four matrices $\mathcal{M}_{A, B, C, D}$ are obtained, based on linearised power equations as described in [9].

\section{Compound Model}

The multi-generator model (figure 3) fits exactly in the structure of equation (6), which is depicted in figure 4.

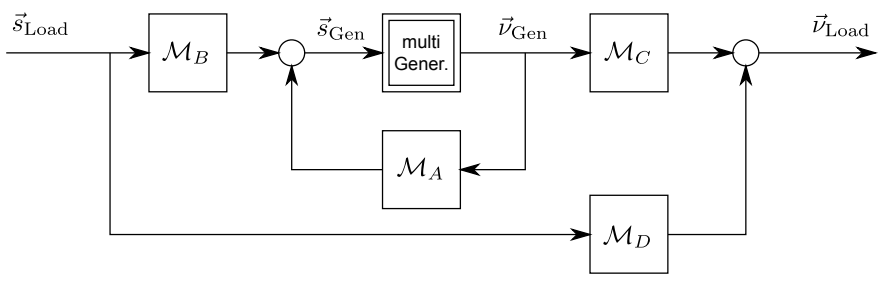

Figure 4. Matrix structure of equation (6)

Inserting the multi-generator model and separating the integrator from the resulting state space model allows for formulating of the final system matrices and the MIMO transfer function matrix $G(s)=\mathcal{C}\left(s \mathcal{I}_{n}-\mathcal{A}\right)^{-1} \mathcal{B}+\mathcal{D}$. An extended explanation of the model can be found in [9].

The presented control model is the first to comprise large island grids with generator dynamics, active and reactive power flows in low voltage grids with ohmic/inductive line characteristics, allowing for stability and dynamics analysis.

\section{GRID DYNAMICS ANALYSIS}

Most results presented in this section, have been obtained from power grid models based on a lattice structure with 16 inverter generators.

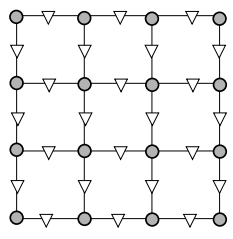

Figure 5. Investigated Lattice Grid Structure

Generators are indicated by a circle, load nodes by a triangle. The lines in between are modelled as low voltage 
cables of equal length $L$, with the characteristics shown in table I.

\begin{tabular}{|c|c|c|c|c|c|}
\hline Level & $U_{0}$ & $S_{0}$ & $L$ & $R^{\prime}$ & $X^{\prime}$ \\
\hline \hline LV & $400 \mathrm{~V}$ & $100 \mathrm{kVA}$ & $500 \mathrm{~m}$ & $0.642 \frac{\Omega}{\mathrm{km}}$ & $0.083 \frac{\Omega}{\mathrm{km}}$ \\
\hline MV & $20 \mathrm{kV}$ & $50 \mathrm{MVA}$ & $10 \mathrm{~km}$ & $0.161 \frac{\Omega}{\mathrm{km}}$ & $0.190 \frac{\Omega}{\mathrm{km}}$ \\
\hline \multicolumn{6}{c}{ Table I } \\
VALUES OF GRID MODEL
\end{tabular}

For the analysis, all inverters have exactly the same individual $5^{\text {th }}$ order model, allowing for basic insights into the system dynamic's dependency on basic inverter model parameters. For all modelled inverters the frequency droop is droopp $=2 \%$, the voltage droop is $\operatorname{droop}_{Q}=4 \%$ and the damping is $k_{D}=0$.

\section{A. Influence of Rated Inverter Power}

The rated power of the inverters has a large influence on the system dynamics. The droop head line is based on each inverter's rated power: the smaller the rated power, the steeper is the headline.
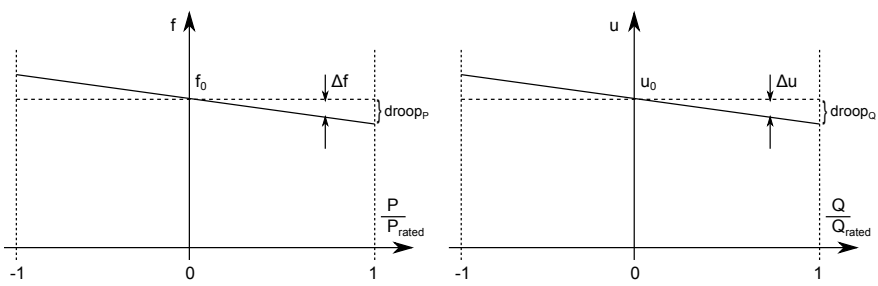

Figure 6. Droop Control Headline

Figure 7 shows pole zero plots for multiple instances of the investigated lattice grid model. Each model has been calculated with a different rated power and its poles and zeros are plotted in a separate colour. The pole positions and colour gradually change with the rated power, as indicated by the colour bar graph on the right.

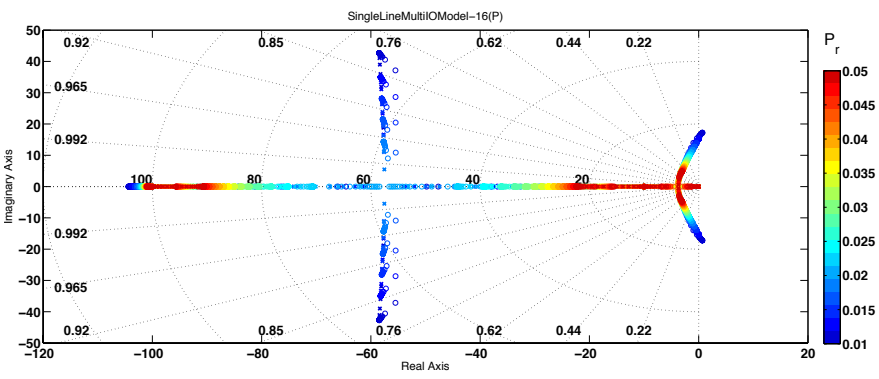

Figure 7. Poles for Models with Different Rated Power

It is visible, that for small rated powers, dominant, conjugate-complex, poorly damped pole pairs appear close to the imaginary axis. These dominant poles move towards and across the imaginary axis for models with lower rated inverter power. A close look at this pole region next to the imaginary axis is revealed in figure 8 .

As each model is calculated with a decreasing rated inverter power, the systems become unstable. The exact stability limit

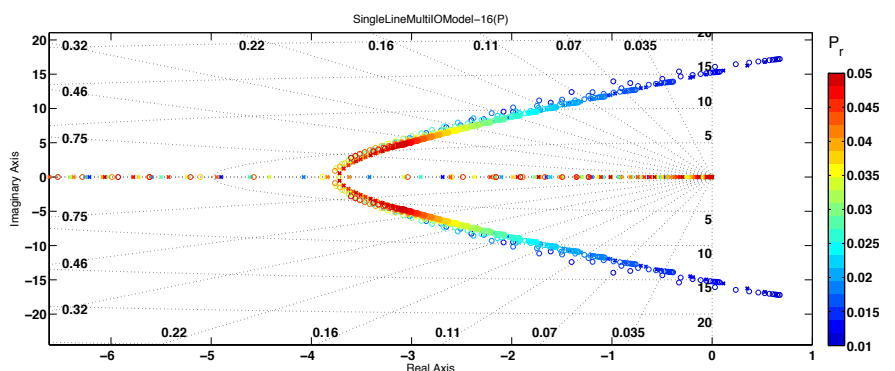

Figure 8. Unstable Poles for Small Rated Power

can be calculated with a robust control toolbox. The physical reason for the instability can be explained: As indicated in figure 6 , the gradient of the droop headline is relative to the rated power:

$$
\dot{\varphi}_{\text {Gen }}=\frac{\text { droop }_{P}}{P_{\text {rated }}} P_{G}+f_{0} .
$$

As the rated power of each inverter is modelled smaller, the droop headline becomes steeper. For a given load situation the inverters react thus more violently with stronger frequency changes. But strong frequency changes lead to large angle drifts and thus to large power flow changes through the grid lines. These, in turn, provoke reactions on all connected inverters and thus the system becomes structurally unstable.

This instability can be compensated by a suitable choice of the generator's lag time constant.

\section{B. Influence of Lag Time Constant}

In the model of figure 2 , the time constant $T_{\mathrm{WR}}$ characterises a $1^{\text {st }}$ order lag. Other than classical rotating generators, who's time constant is bound to the rotor mass inertia, inverters can almost arbitrarily set this time constant in their programming. When inverter time constant are too large, the modelled island grid can become unstable, as figure 9 indicates.

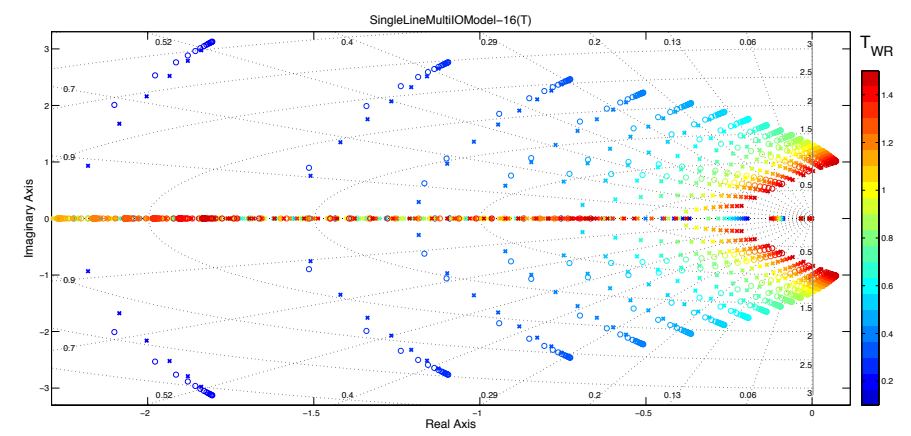

Figure 9. Unstable Poles for Large Time Constants

Physically, this effect is related to the generator's rated power. As has been explained in section III-A, small rated powers lead to violent frequency reactions and to instability. Strong frequency changes in the system can be counteracted by the inverters through a quick reaction. Thus, decreasing the time constant will enable the inverters to more quickly adapt to the new power situation and equilibrate the power flows. The steady state can be reached earlier. This stabilising effect is illustrated in 10 . 


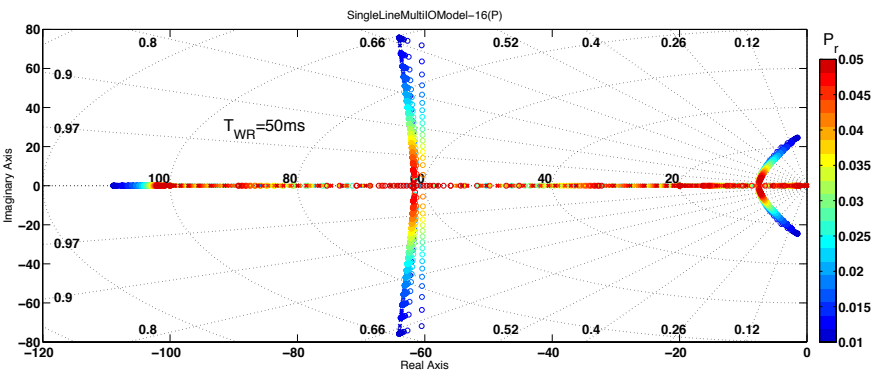

Figure 10. Stabilisation Through Adaptation of Lag Time Constants

Here, inverters have been modelled again with very low rated powers, similar to figure 7 . The main difference is, that in figure 7 a time constant of $T_{\mathrm{WR}}=100 \mathrm{~ms}$ has been used in the model, whereas in figure $10 \mathrm{a}$ time constant of $T_{\mathrm{WR}}=50 \mathrm{~ms}$ has been used. Comparing both illustrations, it becomes visible that by decreasing the time constant $T_{\mathrm{WR}}$, all poles are shifted left, entirely back into the left half pane. Decreasing the time constant stabilises a system with small rated powers.

As a consequence, in inverter driven island grids, the time constant should be proportional to the inverter's rated power.

\section{Influence of Line Length}

For the given model, several instances have been computed with different line lengths. The pole zero plots of the different models are displayed in figure 11 .

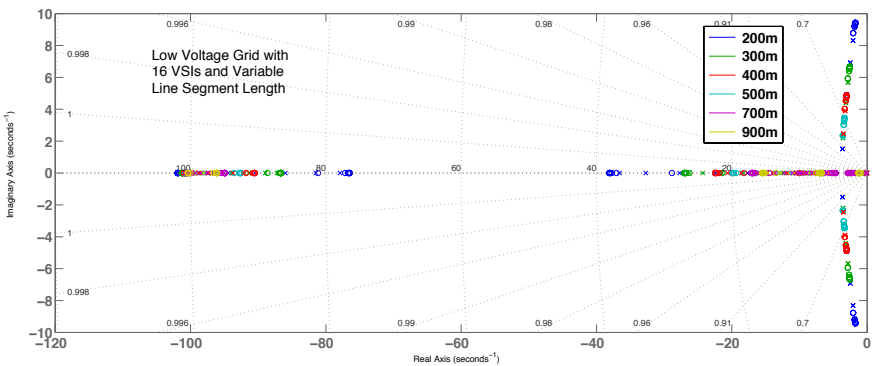

Figure 11. Pole Constellations for Different Line Lengths

The shorter the lines are, the more dominant pole pairs exist close to the imaginary axis. It is visible, that for shorter lines, the poorly damped poles extend further along the imaginary axis. This can mainly be explained by the power flow calculation through the grid lines. The generators adjust voltage angle and amplitude at their points of connection. The phase and amplitude difference between two nodes $i$ and $j$ determine the power flow through the line $k$ between these nodes. The linearised power equation, used in the model is:

$$
\left[\begin{array}{c}
P_{i j} \\
Q_{i j}
\end{array}\right]=\left[\begin{array}{cc}
-B_{k} & G_{k} \\
-G_{k} & -B_{k}
\end{array}\right] \cdot\left[\begin{array}{c}
\delta_{i}-\delta_{j} \\
u_{i}-u_{j}
\end{array}\right],
$$

where $G_{k}$ is the conductivity of the line segment and $B_{k}$ is the susceptibility of the line segment $k$. Decreasing the line length will increase the admittance $Y_{k}=G_{k}+j B_{k}$. For similar angle and amplitude constellations the power flows will be larger for shorter lines. Due to the larger power flows, the frequency and voltage amplitude reactions of the droops will be larger. The generators are thus more closely coupled. The closer dynamic coupling is captured by the model and expressed by more dominant pole pairs.

\section{Influence of $P$-Q-Rotation}

The droop control concept has been developed for high voltage inductive lines: There the active power flow can be controlled through the generator frequency and the reactive power flow can be independently controlled through the generator's voltage amplitude. That is because the resistive part of high voltage lines can be neglected. This is no longer the case in medium or low voltage grids, where the lines have an ohmic-inductive characteristic. As a consequence, a frequency change will not only provoke a change in active power flow, but also influence the reactive power flow. Vice versa, a voltage amplitude change will not only cause a reactive power change but will also influence the active power flow. Thus in ohmic-inductive grids there is a cross coupling effect between active and reactive power. The cross coupling can be partly counteracted by rotating the P-Q-coordinates in the generator before feeding them in the droop control. The compensatory scheme has been described in [10] and shall not be repeated here. This section sheds light on the dynamic situation of the island grids when power rotation is introduced in the generator control.

For the following investigations, the model of a line grid has been built, as indicated by figure 12 . Depending on the

$$
0-0 \nabla-\nabla-\nabla O-0-0 \nabla 0-\nabla
$$

Figure 12. Line Structure

number of generators in the model, line segments were added according to the values in table I.

Figure 13 shows the pole zero plot of a line grid with 50 inverter generators. The poles of the model in non-rotated power coordinates are displayed in blue, the poles of the PQ-rotated model are displayed in green.

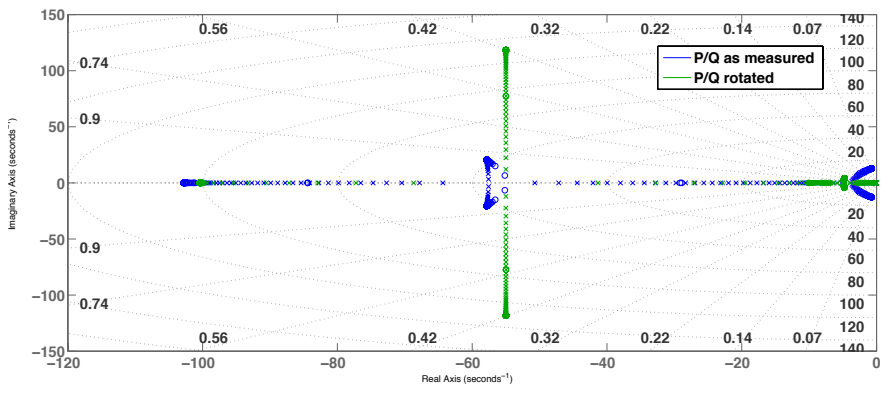

Figure 13. Pole Constellations for Rotated and Non-Rotated Power (LV)

It is visible, that in the centre of the plot, the poles of the power rotated model are less damped and extend further into the complex pane. But more important, close to the imaginary axis, the non-rotated model shows poorly damped, dominant pole pairs close to the imaginary axis. The power rotated model has a higher stability margin.

The influence of the P-Q-rotation on two aspects is investigated: 
1) Medium vs. Low Voltage: Figure 14 shows a comparison between a low voltage grid with power rotation in the generators (blue) and a medium voltage model without power rotation (orange).

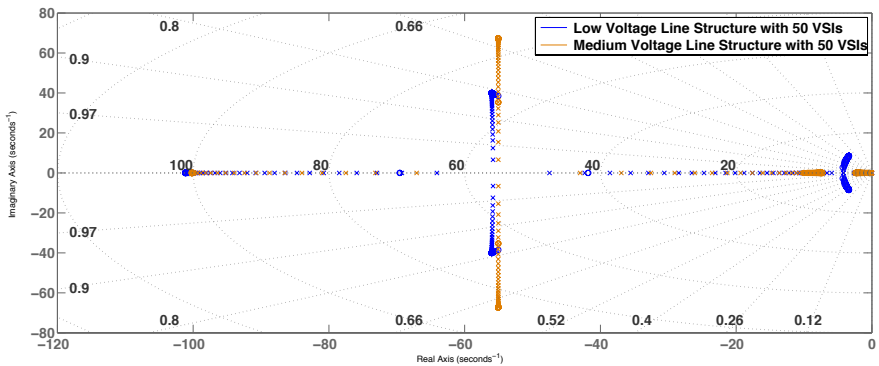

Figure 14. Pole Constellations for Rotated LV grid and Non-Rotated MV grid

As noted in table I, the medium voltage grid has a different line characteristic than the low voltage grid. Due to the more inductive line characteristic, the cross coupling effect is smaller in the medium voltage grid. In figure 14 the medium voltage grid model has no dominant, conjugate complex poles close to the imaginary axis. By power rotation, an ohmic (LV) grid can be better controlled with a (droop) concept developed for inductive grids.

2) Integration Density: Particularly when a high number of inverters is integrated in the grid, the positive effect of the power rotation becomes visible. For a model of a $20 \mathrm{~km} \mathrm{LV}$ line generators are distributed along the line with even spacing. Figure 15 shows the pole zero plot for lines with an increasing number of integrated inverters with power rotation.

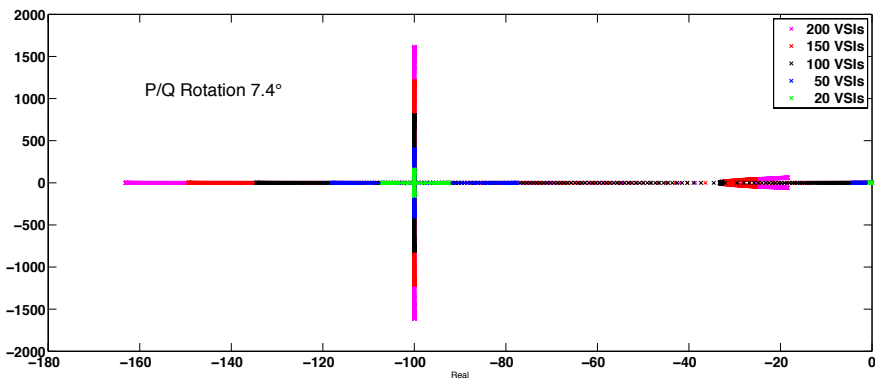

Figure 15. Grids of Inverters with P/Q-Rotation Remain Stable for High Number of Inverters

Only for a very high number of inverters, dominant, conjugate-complex pole pairs appear. Generally all poles remain in the negative real half pane. Figure 16 shows the pole zero plot of similar grid models, but without power rotation in the generator control. Already for low numbers of generators in the system, dominant poles exist. For increasing numbers of generators in the system, these poles move far beyond the imaginary axis into the positive real half pane and the system becomes unstable.

This indicates, that the power rotation enables a much higher density of distributed, renewable power sources in low voltage island grids.

\section{CONCLUSION}

Based on a compound island grid control model, aspects of grid dynamics have been investigated: A pole zero analysis

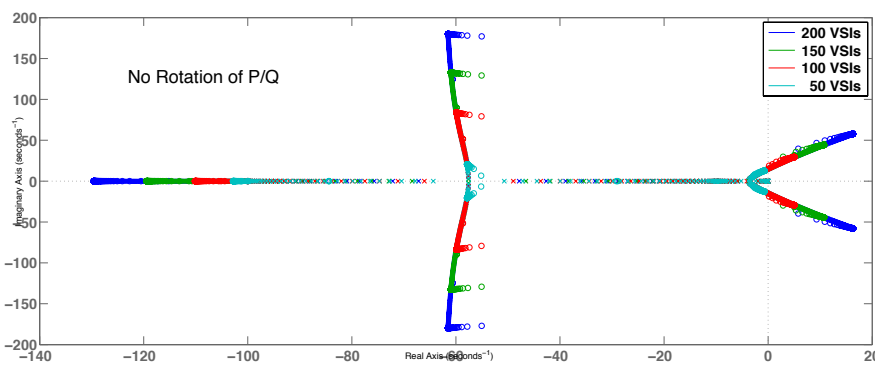

Figure 16. Grids of Inverters without Rotation can Integrate Less Sources

shows that small rated power in conjunction with large time constants can lead to system instability. Close inverter coupling through short grid lines leads to weakly damped, dominant pole pairs. Rotation of P/Q coordinates improves applicability of droop concept in LV lines and allows a higher integration density of inverters before stability limits are reached.

This work is supported by the National Research Fund of

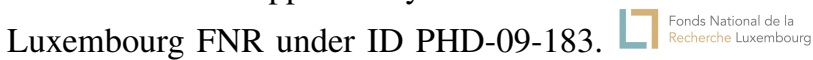

\section{REFERENCES}

[1] M. Jostock and J. Sachau, "Compound model of inverter driven grids," 5th Int. Conf. on Integration of Renewable and Distributed Energy Resources, pp. 194-195, December 2012.

[2] W. Kleinkauf and J. Sachau, "Eurec agency's mega-hybrid project: Electric system technology for renewable energy stand alone applications in the frame of the joule ii programme," EUREC Newsletter, vol. $1,1994$.

[3] J. S. et.al., "Control of parallel working power units in expandable grids," in Proceedings of the 14th European PV Solar Energy Conference and Exhibition, (Barcelona), 1997.

[4] A. Engler and J. Sachau, "Static and rotating grid formation for modularly and expandable island grids," in Proceedings of EPE Conference Lausanne, Switzerland, 1999.

[5] I. Hiskens and E. Fleming, "Control of inverter-connected sources in autonomous microgrids," 2008 American Control Conference, pp. 586-590, June 2008.

[6] A. G. Ritwik Majumber, Balarko Chaudhuri, "Improvement of stability and load sharing in an autonomous microgrid using supplementary droop control," IEEE Transactions on Power Systems, vol. 25, pp. 796-808, May 2010.

[7] A. Engler, Regelung von Batteriestromrichtern in modularen und erweiterbaren Inselnetzen. PhD thesis, Uni Kassel, May 2001.

[8] A. Engler, "Applicability of droops in low voltage grids," DER Journal, January 2005.

[9] M. Jostock, Stability of Purely Inverter Driven Island Power Grids. Phd thesis, University of Luxembourg, 2013.

[10] K. DeBrabandere and $R$. Belmans, " $A$ voltage and frequency droop control method for parallel inverters," in 35th IEEE Power Electronics Specialists Conf., pp. 2501-2507, 2004.

A uthors

Markus Jostock finished his $\mathrm{PhD}$ at the Interdisciplinary Centre for Security, Reliability and Trust (SnT) at the University of Luxembourg, about modelling and stability analysis of purely inverter driven island grids.

Jürgen Sachau is professor and head of the Netpower lab of SnT in DERlab. His research comprises large scale integration of decentral renewable energy resources into the power grid, smart grids and new grid protection.

Jean-Régis Hadji-Minaglou is professor for power electronics at the University of Luxembourg, investigating inverter control modes, motor/generator control and modern power electronics control.

Christian Tuttas is senior researcher at the Institute of Control Systems at the University of Kaiserslautern, Germany. His research domain is in electrical power grids, grid modelling and control and simulation of power electronic devices (FACTS) in power grids. 